\title{
Routine histopathological examination of the foreskin after circumcision for clinically suspected Lichen sclerosus in children: Is it a waste of resources?
}

\author{
Fahad A. Alyami ${ }^{1,2}$; Zhoobin Heidari Bateni ${ }^{1}$; Raken Odeh $^{1}$; Walid A. Farhat ${ }^{1}$; Martin Koyle ${ }^{1}$ \\ ${ }^{1}$ Division of Urology, The Hospital for Sick Children and University of Toronto, Toronto, ON, Canada; ${ }^{2}$ Urology \\ Division, Department of Surgery, King Saud University, College of Medicine, King Saud University Medical City, \\ Riyadh, Saudi Arabia
}

Cite as: Can Urol Assoc J 2018 Feb. 6; Epub ahead of print. http://dx.doi.org/10.5489/cuaj.4331

Published online February 6, 2018

$* * *$

\section{Abstract}

Introduction: Circumcision is one of the most widely performed procedures in the world. One of the indications for circumcision is Lichen sclerosis (LS). The natural history of LS in children is not as well-documented as in adult patients. Surgeons use the appearance of the foreskin or meatus to predict the diagnosis of LS. ${ }^{1}$ Indeed, if the diagnosis of LS is made in childhood, does it change management in the long term? Pathological analysis of the excised foreskin is routinely done if there is suspicion of LS. Our aim is to assess the concordance between the clinical and pathological diagnosis of suspected LS and to assess the need for sending the foreskin for pathological examination.

Methods: We conducted a retrospective chart review of 64 of 420 boys who underwent circumcision in a tertiary children's hospital from June 2005 to June 2014, and who had their foreskin sent for pathology due to the clinical suspicion of LS. Demographics, presenting symptoms, presumed clinical diagnosis, and pathological findings and followup data were collected and analyzed.

Results: Over the review period, 64 patients underwent circumcision for presumed LS. The mean age of the children was 9.7 years (3-16.5). All the children who had circumcision for presumed LS diagnosis were symptomatic. LS was confirmed in 47 of 64 foreskins (73.5\%). BXO was clinically suspected in 40 (85\%) of the 47 patients. The mean followup was 10 months (1-15) with seven recurrences (15\%) during that period. The recurrences required revision surgery in two patients and five were managed with steroids only.

Conclusions: In our series, the clinical diagnosis correlated with the pathological diagnosis in most cases. A clinical suspicion of LS without routine foreskin pathological assessment will reduces the overall cost to the healthcare system. Appropriate counselling of the patient/parents and their primary caregiver is imperative, as recurrence is common. 


\section{Introduction}

Circumcision is one of the most widely practiced procedures in the world. It is mostly happening for religious or cultural and sometimes therapeutic reasons. ${ }^{2}$ One of the unique medical indications for circumcision is Lichen sclerosis (LS). LS is a chronic progressive inflammatory disease of the male external genitalia area mostly involving glans penis, meatus and prepuce which first described by Stuhmer in $1928 .^{3}$

The natural history of the disease in adults is well documented. Progression of the disease can cause meatal stenosis, urethral stricture and and may be a precursor to Squamous Cell Carcinoma of the penis. ${ }^{3,4,7}$ Despite the established relationship between natural history,clinical findings and pathological diagnosis of LS in adults, this relationship is not well documented in children. 5,6, 8, 9,10,11

In a prospective study of 1178 boys with phimosis, Kiss et al found the incidence of LS was $40 \%$. LS can present at any age but the most common age in children between 9 to 11 years of age. In this study they found that phimosis occurred in $93 \%$ of the patients with LS and only in $32 \%$ without $\mathrm{LS}^{6}{ }^{6}$ In a recently published study, Celis et al could not find a correlation between clinical and pathological diagnosis. ${ }^{11}$

The appearance of the prepuce, meatus or glans penis at circumcision for LS has been used by surgeons to predict the pathological diagnosis. With increased health care costs due to unnecessary studies and knowing that routine pathological assessment of the foreskin after circumcision is not always mandated, we aimed to assess the correlation between the clinical and pathological diagnosis and to assess the need for sending the foreskin for pathological examination.

\section{Methods}

\section{Patients}

After institutional ethics board approval we reviewed the medical records of all boys who underwent to circumcision by different surgeons in a single tertiary children hospital between June 2005 to June 2014. Only those whose foreskins were sent for histopathological examination due to suspicion of LS were included for analysis.

Information obtained from clinic visit and operative notes and were reviewed for: age at first presentation of symptoms, circumcision status, relevant presenting symptoms (ballooning of prepuce, spraying during urination, straining, frequency, urgency, urinary retention, balanitis and urinary tract infection.), previous medical treatments, intraoperative and post-operative management, description of the lesions in operation room under anesthesia and clinical suspicion of LS, subsequent outcomes and follow up. Data were analyzed using SPSS version 15.0 software (SPSS Inc., Chicago, IL). The total financial costs of sending the suspicious prepuce specimens for the pathologic evaluation was evaluated. 


\section{Results}

During the period of analysis, 420 circumcisions were performed of which 64 (15\%) had foreskins pathological assessment due to suspicion of LS. The mean age of children was 10 (range 3.5-16.5) years. All the children who had circumcision for presumed LS diagnosis were symptomatic: 48 (75\%) ballooning of the foreskin, 42 (66\%) straining, 28 (44\%) spraying, , 9 (14\%) urinary retention, 9 (14\%) history of UTIs and 23 (36\%) had recurrent balanoposthitis. Out of the 64 patients 33 patients failed medical treatment (4-6 weeks of topical steroids then ended up with circumscion.

LS was confirmed in 47 out of 64 foreskins (73.5\%). BXO was clinically suspected in 40 (85\%) out of the 47 patients. Chronic inflammation was reported in 15 (23.5\%) patients and normal foreskin was found in only 2 (3\%) patients. The mean follow up time was 10 months (1 15 months) with 7 recurrences (15\%) during that period. The recurrences required revision surgery in 2 patients and 5 were managed with steroids only.

In our series the cost for each pathological exam ranges from \$125 to \$200 CAD. The estimated total cost for the pathological examination for the 64 patients was \$10000 CAD.

\section{Discussion}

In young boys LS is a chronic inflammatory disease which involves prepuce, urethral meatus and glans of the penis with unknown etiology and an uncertain but definite propensity to recur. In our population with presumed LS, $76 \%$ were confirmed histologically and only $2 \%$ had normal histology. This confirms that in most instances, there is excellent correlation between clinical suspicion and ultimate pathology of LS and other inflammatory lesions, and begs the question of the necessity of pathological confirmation.

In a recent epidemiological study the incidence of LS per year was near 0.03 percent in boys under 5 years of age, almost 10 times higher than in boys under 15 years of age. ${ }^{12}$ It seems that there is an inevitable need for conducting more studies on LS, potentially on a larger scale in children and more specifically in boys to find all aspects of this health related issues of these chronic lifelong diseases. The incidence of LS ranges between 5 to more than 50 percent of all patients who have undergone surgery due to phimosis, the higher percentages found in more recent studies. ${ }^{5,6,13}$ In an era of restricted resources, coupled with the clinical suspicion, the value of routine pathological assessment of the clinically suspected LS foreskin may need to be revisited.

There is controversy in the literature between the similarities of clinical and pathological diagnosis of the LS, despite the clinicopathological correlation in our series. In our study, most of the foreskins sent for pathology were for patients with a history of recurrent balanoposthitis, phimosis or suspicious dermatological lesions. In our province in Canada, non-medically indicated circumcision is not covered by our single payer system. Hence, our study identifies a highly selected, referral population who presented with a history of certain symptoms, such as difficulty in urination, ballooning of the foreskin, history of phimosis, urinary tract infections and suspicious lesions of prepuce. Results showed that all our patients with clinically suspected LS 
ended up needing circumcision , 33 (51\%)failed medical treatment and the rest needed upfront circumcision. We don't send foreskin for pathological assessment routinely, unless a pathological diagnosis is suspected with hopes of improving postoperative patient counselling.

In our study we found an $85 \%$ correlation between clinical and pathological diagnosis of LS, which was similar to other studies found in the literature ${ }^{5,8,12}$. Yardley et al, found that 88.9\% of their patients with the final pathological diagnosis of LS had presented with suspicious clinical symptoms in an outpatient clinic before the surgery. ${ }^{11}$ Based on the high correlation we found in our study after circumcision, we suggest to avoid sending all suspicious circumcision samples to pathology, but rather counsel the parents/patients and caregivers, as to the presumed diagnosis of LS, and accordingly and discuss the signs and symptoms of recurrence.

In our provincial health system, routine submission of the excised prepuce after circumcision is no longer mandated. Although submitting all tissue may provide findings not suspected clinically, the likelihood of finding an abnormality in an otherwise healthy child is minute (14). Thus a limitation of this paper is that by not sending all foreskins, ie the other 354, might we have missed other cases of LS where there was no clinical suspicion. Clinical suspicion however, can be wrong in over, as well as under diagnosing pathology. We acknowledge that this retrospective study focuses on a select group of patients where there was suspicion of pathology. Given the spectrum regarding the natural history of LS, with the majority of cases being non-progressive by performing circumcision alone (15) one must question the value of submitting the foreskin when LS is suspected, rather than counseling and educating patients and families appropriately.as to the gross abnormality of the foreskin and the potential small risk for subsequent abnormalities.

We acknowledge important limitations of our study, including its retrospective nature, the focus on a selected small group of patients. Other studies have revealed a weaker correlation between suspicious clinical findings and pathological confirmation of the LS. ${ }^{9,11}$ We believe that most of these variances could be due to observer differences and again to the fact over our referral bias in the Canadian system. Therefore, there is value in conducting a prospective study with a large number of patients with solid clinical criteria to then find a more reliable percentage of this correlation, which could be helpful to provide better management and care for these patients at initial presentation.

Based on our observation, there is a possibility that 1 out of 10 patients are falsely diagnosed as clinically suspicious LS. This false positive diagnosis would not have any adverse effect on the history of the LS treatment for that patient or any significant financial burden on the health care system, as this misleading diagnosis is negligible.

\section{Conclusion}

Clinical vs. pathological diagnosis of LS, if considered based on the clinical observation of an expert pediatric urologist could have a good concordance rate eliminating the routine pathological evaluation. In an era of financial constraints judicious use of the resource including 
relying on the physical exam to predict the diagnosis of LS may translate into a more costeffective use of time and resources.

\section{References}

1. Holbrook $\mathrm{C}^{1}$, Tsang T. Management of boys with abnormal appearance of meatus at circumcision for balanitis xerotica obliterans. Ann R Coll Surg Engl. 2011 Sep;93(6):482-4.

2. Demaria J, Abdulla A, Pemberton, J et al. Are physicians performing neonatal circumcisions well-trained? Can Urol Assoc J 2013;7(7-8):260-4.

3. Das S, Tunuguntla HS. Balanitis xerotica obliterans--a review. World J Urol 2000;18:382-7.

4. Philippou P, Shabbir M, Ralph DJ, et al. Genital lichen sclerosus/balanitis xerotica obliterans in men with penile carcinoma: a critical analysis. BJU Int. 2013;111(6):970-6

5. Gargollo PC, Kozakewich HP, Bauer SB, et al. Balanitis xerotica obliterans in boys. J Urol. 2005;174(4 Pt 1):1409-12.

6. Kiss A, Király L, Kutasy B, et al. High incidence of balanitis xerotica obliterans in boys with phimosis: prospective 10-year study. Pediatr Dermatol. 2005;22(4):305-8.

7. Clouston D, Hall A, Lawrentschuk N. Penile lichen sclerosus (balanitis xerotica obliterans). BJU Int. 2011;108(Suppl 2):14-9.

8. Vincent MV, Mackinnon E. The response of clinical balanitis xerotica obliterans to the application of topical steroid-based creams. J Pediatr Surg. 2005;40(4):709-12.

9. Kuehhas FE, Miernik A, Weibl P, et al. Incidence of balanitis xerotica obliterans in boys younger than 10 years presenting with phimosis. Urol Int. 2013;90(4):439-42.

10. Mattioli G, Repetto P, Carlini C, et al. Lichen sclerosus et atrophicus in children with phimosis and hypospadias. Pediatr Surg Int. 2002;18(4):273-5.

11. Celis S, Reed F, Murphy F, et al. Balanitis xerotica obliterans in children and adolescents: a literature review and clinical series. J Pediatr Urol. 2014 Feb;10(1):34-9.

12. Yardley IE, Cosgrove C, Lambert AW. Paediatric preputial pathology: are we circumcising enough? Ann R Coll Surg Engl. 2007;89(1):62-5

13. Jayakumar S, Antao B, Bevington O, et al. Balanitis xerotica obliterans in children and its incidence under the age of 5 years. J Pediatr Urol 2012; 8: 272-275.

14. Naji H, Jawad E, Ahmed HA, et al. Histopathological examination of the prepuce after circumcision: Is it a waste of resources? Afr J Paediatr Surg. 2013 AprJun;10(2):164-6.

15. Mohammed A, Shegil IS, Christou D, et al. Paediatric balanitis xerotica obliterans: an 8year experience. Arch Ital Urol Androl. 2012 Mar;84(1):12-6. 